\title{
MINDFULNESS Y ANSIEDAD EN PACIENTES CON OBESIDAD MÓRBIDA
}

\author{
Erin Yaqueline Tovar Garza, Luz Adriana Orozco Ramírez y José Luis Ybarra Sagarduy \\ Universidad Autónoma de Tamaulipas \\ México
}

\begin{abstract}
RESUMEN
El deterioro en la salud y discriminación consecuentes a la obesidad contribuyen a que aparezcan trastornos de ansiedad. Una técnica que ha mostrado su efectividad en la ansiedad es el Mindfulness, la cual consiste en la aceptación de emociones, cogniciones y sensaciones, mas no su modificación. El propósito del presente estudio es explorar las asociaciones entre las facetas de mindfulness y las dimensiones de la ansiedad en población adulta con obesidad mórbida. Es un estudio exploratorio ex post facto retrospectivo de un grupo con múltiples medidas. La muestra fue no probabilística por cuota, con siete pacientes de una clínica de obesidad en Ciudad Victoria. Los resultados mostraron una importante correlación negativa entre la faceta de mindfulness no reactividad ante las experiencias y la respuesta de ansiedad motora, al igual con las situaciones ansiógenas de evaluación, interpersonal y de la vida cotidiana. Estos datos sugieren que en intervenciones basadas en mindfulnees para pacientes con obesidad sea importante considerar estrategias del control de la no reactividad y la ansiedad.
\end{abstract}

\section{Palabras Clave:}

mindfulness, respuestas de ansiedad, situaciones de ansiedad, obesidad mórbida, facetas de mindfulness, no-reactividad

\section{MINDFULNESS AND ANXIETY IN PATIENTS WITH MORBID OBESITY}

\begin{abstract}
The deterioration in health and discrimination resulting from obesity contribute to anxiety disorders. One technique that has shown its effectiveness in anxiety is Mindfulness, which consists in the acceptance of emotions, cognitions and sensations, but not its modification. The purpose of the present study was to explore the associations between the facets of mindfulness and the dimensions of anxiety in the adult population with morbid obesity. This is an ex post facto retrospective exploratory study of a group with multiple measures. The sample was not probabilistic by quota taking 7 patients from an Obesity Clinic in Ciudad Victoria. The results showed that there is a strong negative correlation between the facet of mindfulness not reactivity to the experiences and the response of motor anxiety, as well as the anxious situations of evaluation, interpersonal and everyday life. These data suggest that in mindfulne-based interventions for patients with obesity, it is important to consider non-reactivity and anxiety management strategies.
\end{abstract}

Keywords:

mindfulness, anxiety responses, anxiety situations, morbid obesity, facets of mindfulness, non-reactivity

Bitácora del Artículo:

| Recibido: 1 de Junio de 2019 | Aceptado: 1 Noviembre de 2019 | Publicado en línea: Enero-Junio de 2020 | 
Artículo Empírico | Mindfulness y ansiedad en pacientes...| Tovar-Garza, Orozco-Ramírez e Ybarra-Sagarduy

\title{
Autoría y Derechos de Propiedad Intelectual
}

\section{MINDFULNESS Y ANSIEDAD EN PACIENTES CON OBESIDAD MÓRBIDA}

\author{
Erin Yaqueline Tovar Garza, Luz Adriana Orozco Ramírez y José Luis Ybarra Sagarduy \\ Universidad Autónoma de Tamaulipas \\ México
}

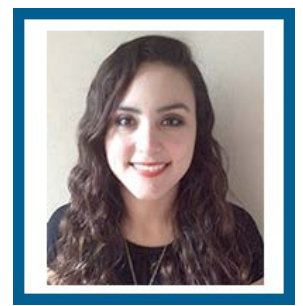

Erin Yaqueline Tovar Garza

Universidad Autónoma de Tamaulipas

Correo: erintovar94@gmail.com

Licenciada en Psicología por la Universidad La Salle Victoria (ULSA). Actualmente está cursando la maestría en Psicología Clínica y de la Salud en la Universidad Autónoma de Tamaulipas (UAT). Es miembro activo del Colegio de Psicólogos Victorense.

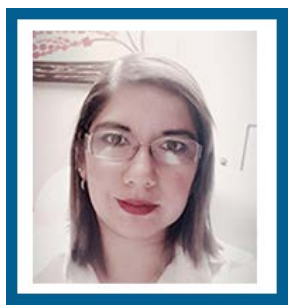

Luz Adriana Orozco Ramírez Universidad Autónoma de Tamaulipas Correo: adyluz21@gmail.com

Doctora en Psicología por la Universidad de Sevilla, España. Es Docente investigador de tiempo completo categoría " $D$ " en la Universidad Autónoma de Tamaulipas (UAT). Líder del Cuerpo Académico en Consolidación de Estudios de Psicología (CAEP)..

\section{CONTRIBUCIÓN DE LOS AUTORES}

Erin Yaqueline Tovar Garza: Concibió la idea, diseño y desarrollo del proyecto, llevó a cabo el trabajo de campo, participó en el análisis de resultados y en la elaboración del manuscrito | Luz Adriana Orozco Ramírez: Hizo el análisis de datos, desarrolló el proyecto y elaboró el manuscrito | José Luis Ybarra Sagarduy: Hizo el análisis de datos, desarrollo el proyecto y elaboró el manuscrito

\section{AGRADECIMIENTOS}

A la clínica de obesidad del Hospital Regional de Alta Especialidad de Ciudad Victoria y al programa de Maestría en psicología clínica y de la salud de la Universidad Autónoma de Tamaulipas, quienes a través de su apoyo hicieron posible el desarrollo de la investigación.

\section{DAtos de Filiación de los Autores}

Universidad Autónoma de Tamaulipas

\section{(2) $(1) \Theta(0)$}

Copyright: (c) 2020 Tovar-Garza, E. Y., Orozco-Ramírez, L. A. \& Ybarra-Sagarduy, J. L.

Este es un artículo de acceso abierto distribuido bajo los términos de la licencia Creative Commons Reconocimiento-NoComercial 4.0 Internacional, por lo que su contenido gráfico y escrito se puede compartir, copiar y redistribuir total o parcialmente sin necesidad de permiso expreso de sus autoras con la única condición de que no se puede usar con fines directamente comerciales y los términos legales de cualquier trabajo derivado deben ser los mismos que se expresan en la presente declaración. La única condición es que se cite la fuente con referencia a la Revista Digital Internacional de Psicología y Ciencia Social y a sus autoras. 


\section{TABLA DE CONTENIDO}

MÉTODO

Diseño, 264

Participantes, 264

Materiales, 264

Procedimiento, 265

Análisis estadísticos, 265

REsultados

Discusión

CONCLUSIONES

REFERENCIAS 


\section{L} a obesidad es una enfermedad sistémica, crónica y multicausal, no exclusiva de países económicamenedad, etnias y clases sociales (Organización Mundial de la Salud, 2014). En un análisis reciente de la transición epidemiológica en México se reveló que las enfermedades propiciadas por la obesidad causaron $75 \%$ del total de las muertes y $68 \%$ de los años de vida potencialmente perdidos (Barrera, Rodríguez y Molina, 2013).

Se clasifica en distintos grados, los cuales determinan los factores de riesgo, pronósticos y la calidad y esperanza de vida; el más avanzado de ellos es la obesidad mórbida, la cual es una condición de salud grave que puede interferir con las funciones físicas básicas, como respirar o caminar; se diagnostica cuando el índice de masa corporal (IMC) es de 40 o más (Secretaría de Salud, 2012).

Además de los efectos en la salud física, la obesidad también se asocia con mayores índices de depresión y ansiedad, los cuales impactan en la adherencia al tratamiento y los resultados de las intervenciones para la pérdida de peso (Rogers, Ferrari, Mosely, Lang y Brennan, 2016), por lo que trabajar con la reducción de la ansiedad es de utilidad para la efectividad del tratamiento.

La ansiedad suele estar unida a una importante activación fisiológica y de comportamiento. La intensidad de dichas manifestaciones de ansiedad depende de factores situacionales (intensidad de la situación, novedad, etcétera), de factores personales (rasgo de ansiedad, tendencias personales, etcétera), así como la interacción de ambos tipos de factores (Schmidt y Vinet, 2015).

El modelo interactivo de la ansiedad (Endler, 1981) insiste en la necesidad de considerar la interacción de ambos factores, porque la evaluación del rasgo de personalidad, independientemente de las situaciones, es muy general.

Por tanto, es necesario evaluar los distintos tipos de respuesta de ansiedad (cognitivo, fisiológico y motor) en las situaciones potencialmente ansiógenas (ansiedad de evaluación, interpersonal, fóbica y ansiedad en la vida cotidiana), para determinar qué situaciones perturban más al sujeto, así como para determinar cuál es su respuesta hacia ellas (Cano-Vindel, Miguel-Tobal, Gonzalez e Iruarrizaga, 1994).

Se hablaría de reactividad individual de tipo cognitivo, fisiológico o motor, según la que predomine. Esta alta especificidad individual (reactividad) estaría relacionada con una eficacia diferente de las técnicas de tratamiento; si un sujeto presenta un tipo de reactividad marcada (ya sea cognitiva, fisiológica o motora) se debería comenzar el tratamiento con una técnica de tipo concordante con su reactividad (Cano-Vindel et al., 1994).

Una de las técnicas que se utilizan para tratar la ansiedad es el Mindfulness o Atención Plena; ésta se integra en las terapias de tercera generación (Baer, Hopkins, Krietemeyer, Smith y Toney, 2006) y es típicamente definido como el proceso de atención plena a la experiencia del momento presente sin prejuicios (Bishop et al., 2004; Kabat-Zinn, 1990).

Los tratamientos basados en mindfulness incluyen varias técnicas basadas en las facetas teorizadas de la atención plena (Holzel et al., 2011); dichas facetas influyen en la salud psicológica de diferentes maneras. Por tanto, es importante determinar, al igual que con la situaciones y respuestas de ansiedad, qué facetas son influencias positivas, porque éstas pueden promoverse en la población que se pretende atender (Tomlinson, Yousaf, Vittersø y Jones, 2017).

Aunque varios investigadores, como Keng, Smoski y Robins (2011), Kocovski, Segal, Battista y Didonna (2009) y Teasdale, Segal y Williams (2003), han abogado por una aplicación más específica de técnicas de atención plena, se sabe poco acerca de cómo afectan las facetas del mindfulness en la ansiedad (Keng, Smoski y Robins, 2011). Un estudio - Baer et al. (2006), a través de un análisis de regresión- encontró que sólo las facetas "actuar con conciencia", "no juzgar" y "no reactividad" eran predictores significativos de síntomas psicológicos.

Por otra parte, los estudios de Tomlinson et al. (2017) y Medvedev, Norden, Krägeloh y Siegertet (2018) analizaron qué facetas específicas pueden predecir la ansiedad en poblaciones no clínicas; los resultados indicaron que niveles más bajos de autojuicio resultaban en puntuaciones más bajas de ansiedad, por lo que se ha llegado a sugerir que el desarrollo de la faceta "no juzgar" debe considerarse como el factor más importante para la reducción de la ansiedad (Medvedev et al., 2018).

Sin embargo estos resultados se obtuvieron en una población no clínica, por lo que es necesario analizar si las relaciones entre las facetas de mindfulness y la ansiedad se relacionan de la misma manera en una población con obesidad.

Por tanto, el propósito del estudio es identificar las situaciones y respuestas de ansiedad predominantes, la faceta de mindfulness con mayor presencia, y las relaciones entre los niveles de mindfulness y ansiedad general, las facetas de mindfulness y las situaciones de ansiedad, y entre las facetas de mindfulness y las res- 
puestas de ansiedad, en una población de pacientes con obesidad mórbida.

Partiendo de la hipótesis de que, de manera similar a los estudios efectuados en poblaciones no clínicas, la faceta "no juzgar" mostrara mayor relación con las situaciones y respuestas ansiedad en una población clínica como los pacientes con obesidad mórbida.

\section{MÉTodo}

\section{Diseño}

Se hizo un estudio exploratorio ex post facto retrospectivo con un solo grupo (Montero y León, 2007), donde se intentó analizar la relación de las respuestas y las situaciones de ansiedad con las facetas del mindfulness en un grupo único de pacientes con obesidad mórbida.

\section{Participantes}

Se utilizó un muestreo no probabilístico consecutivo con pacientes del Hospital Regional de Alta Especialidad de Ciudad Victoria (HRAEV) pertenecientes a la clínica de obesidad en el periodo de marzo-mayo de 2016. Entre los criterios de inclusión se consideró que fueran pacientes que presentaran obesidad mórbida y fueran mayores de edad. El grupo final de participantes constó de siete pacientes (cinco mujeres y dos hombres), con edades de 22 a 50 años.

Se presentan los datos sociodemográficos de los participantes (tabla 1), donde la media de edad de los participantes es de 35.86, siendo $58 \%(n=4)$ mujeres y $42 \%(n=3)$ hombres; la mayoría $(58 \% ; n=4)$ con estado civil soltero, con una media de peso de 135.31, una media de talla 165.43 y una media de IMC de 49.19.

Tabla 1

Datos sociodemográficos de pacientes con obesidad del HRAEV

\begin{tabular}{|c|c|c|c|c|c|c|c|c|}
\hline & & & EDAD & Ocupación & ESTADO CIVIL & Peso & TALLA & IMC \\
\hline \multirow[t]{5}{*}{$F$} & 1 & & 48 & Enfermera & Div & 113.0 & 160 & 44.50 \\
\hline & 2 & & 39 & Ama de casa & Sol & 157.2 & 152 & 67.30 \\
\hline & 3 & & 22 & Estudiante & Sol & 96.3 & 161 & 37.20 \\
\hline & 4 & & 28 & Estilista & Sol & 108.0 & 163 & 40.75 \\
\hline & Total & $\mathrm{N}$ & 4 & 4 & 4 & 4 & 4 & 4 \\
\hline \multirow[t]{4}{*}{ M } & 1 & & 28 & Estudiante & Sol & 173.7 & 180 & 55.90 \\
\hline & 2 & & 40 & Profesor & Uni & 139.0 & 169 & 48.70 \\
\hline & 3 & & 46 & Profesor & Cas & 160.0 & 173 & 50.00 \\
\hline & Total & $N$ & 4 & 3 & 3 & 3 & 3 & 3 \\
\hline
\end{tabular}
cinco facetas de conciencia plena.

1) Observación. Se refiere a darse cuenta o asistir a experiencias internas y externas, como sensaciones, cogniciones, emociones, sonidos y olores (ítems 1, 6, 11, 15, 20, 26, 31 y 36).

2) No juzgar. Se refiere a adoptar una postura no evaluativa hacia pensamientos y sentimientos (ítems $3^{*}, 10^{*}, 14^{*}, 17^{*}, 25^{*}, 30^{*}, 35^{*}$ y $\left.39^{*}\right)$.

3) No reactividad. Se refiere a la tendencia de permitir que los pensamientos y sentimientos fluyan, sin quedar atrapados en o dejarse llevar por ellos (ítems 4, 9, 19, 21, 24, 29 y 33).

4) Descripción. Se refiere a etiquetar experiencias internas con las palabras (ítems 2, 7, 12*, 16*, 22*, 27,32 y 37 ).

5) Actuar consciente. Se refiere a la atención en las actividades del momento y puede ser contrastado con comportarse de manera mecánica mientras la atención se enfoca en otro lugar (piloto automático) (ítems 5*, 8*, 13*, 18*, 23*, 28*, 34* y $38^{*}$ ).

El cuestionario tiene ítems de puntuación inversa y directa que se colocan en una escala de tipo Likert de cinco valores, que van de uno = nunca o muy raramente verdadero a cinco $=$ muy a menudo o siempre verda-
Se aplicó la versión estandarizada en español, la cual recoge información por medio de 39 ítems que conforman

\section{Materiales}

Los participantes completaron una ficha de datos sociodemográficos que incluía género, edad, ocupación, estado civil, peso, talla e IMC.

\section{- Five Facet Mindfulness Questionnaire}

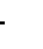


dero. También hay 19 ítems de puntuación inversa $(*)$ (Schmidt y Vinet, 2015).

A la vez se obtiene una puntuación de mindfulness general por medio de la suma de las cinco facetas, donde, al igual que en las subescalas, a mayor puntuación mayor presencia. En el estudio que da origen a la validación del FFMQ (Baer et al., 2006), tuvo una buena consistencia interna en sus subescalas (observar $=0.83$, describir $=0.91$, actuar con conciencia $=0.87$, no enjuiciar $=0.87$ y no-reactividad $=0.75)$, así como una buena consistencia en la escala total (0.87).

Por su parte, Meda, Herrero, Blanco, Moreno-Jimenez y Palomera (2015) validaron el cuestionario en población mexicana, el cual mostró índices de consistencia interna buenos para todas las subescalas del FFMQ (observar $=0.82$, describir $=0.84$, actuar con conciencia $=0.70$, no juzgar $=$ 0.61 y no-reactividad $=0.73)$, y en la escala total $(0.70)$.

\section{- Inventario de situaciones y respuestas de ansiedad (ISRA) de Tobal y Vindel (1999)}

El inventario de situaciones y respuestas de ansiedad (ISRA) permite la evaluación por separado de los tres sistemas de respuesta de ansiedad.

1) Cognitiva. Se refiere a pensamientos y sentimientos de preocupación e inseguridad (23 ítems).

2) Fisiológica. Agrupa las respuestas que muestran la activación del sistema nervioso autónomo y otros síntomas, como dolor de cabeza (23 ítems).

3) Motora. Se refiere a síntomas de ansiedad observables, como tartamudez o llanto (23 ítems).

Por otro lado, también permite la evaluación de la ansiedad asociada a cuatro áreas situacionales o rasgos específicos.

1) Ansiedad de evaluación. Engloba situaciones de evaluación o asunción de responsabilidades, como hablar en público (ítems 1, 4, 8, 10, 11 y 13).

2) Ansiedad interpersonal. Se refiere a situaciones interpersonales, como una situación sexual íntima o asistir a una reunión social (ítems 7, 15 y 18).

3) Ansiedad fóbica. Agrupa situaciones potencialmente fóbicas, como viajar en avión (ítems 12, 14, 17 y 19 ).

a) Ansiedad en la vida cotidiana. Comprende situaciones cotidianas, como la hora de dormir (ítems: 5,21 y 22 ).

Consta de 224 ítems, con escala Likert de 0 (casi nunca) a 4 (casi siempre) que evalúa la frecuencia con que se presenta una serie de respuestas características de la ansiedad (24 respuestas) ante situaciones concretas (22 situaciones). Para la interpretación de las puntuaciones, éstas se convertirán en percentiles utilizando baremos según la naturaleza de la población (clínica o no clínica), donde a mayor puntuación mayor presencia de la variable.

La consistencia interna en población mexicana para el total de los ítems de la escala es de 0.94, para las tres subescalas de respuestas 0.90 (cognitivas 0.83, fisiológicas 0.86 y motoras 0.69) y 0.89 para las cuatro subescalas de áreas situacionales (evaluación 0.81, interpersonales 0.63, fóbicas 0.78 y cotidianas 0.61 ); tiene una fiabilidad test-retest de 0.78 (Cano-Vindel, Fernández y Spielberger, 2012).

\section{Procedimiento}

Las pruebas se aplicaron de manera individual por el investigador, por lo que cada participante fue citado a distinta hora; ambos instrumentos se aplicaron en el área de psicología durante la sesión de evaluación, la cual tiene lugar en la primera sesión a la que asisten los participantes. Se informó a los participantes de la muestra individualmente y de manera verbal los objetivos de la investigación, y mediante un consentimiento informado se indicó la finalidad de la investigación y del instrumento, así como que la información sería manejada de manera confidencial y ética con fines de investigación; ellos decidían participar o no en la misma.

Se comenzó con la aplicación del test ISRA, el cual tiene una duración de 40 a 50 minutos; esta prueba tuvo una modalidad autoaplicada con el investigador presente para resolver cualquier duda que surgiera. Cuando terminó la aplicación del test ISRA se continuó con el FFQM, el cual tuvo una modalidad autoaplicada donde, de igual manera que en la prueba anterior, estuvo presente el investigador en caso de que se presentaran dudas; esta prueba tuvo una duración de casi 20 minutos.

\section{Análisis estadísticos}

Para el procesamiento de la información se utilizó el paquete estadístico SPSS 21. Para la estadística descriptiva se consideraron los resultados de la moda, desviación típica o estándar, y las puntuaciones mínimas y máximas de las medidas antropométricas. Para la correlación de las puntuaciones de las variables de ansiedad y mindfulness se utilizó la prueba no paramétrica del coeficiente de correlación de Spearman, $\rho$ (rho).

\section{Resultados}

\section{Respuestas y situaciones de ansiedad predominantes en pacientes con obesidad mórbida}

Durante el análisis del tipo de respuestas a la ansiedad en los pacientes con obesidad mórbida (tabla 2) se encontró 
que hay una mayor presencia de la ansiedad fisiológica, la cual se refiere a los cambios corporales; éstos se caracterizan por una elevada activación, sobre todo del sistema nervioso autónomo (sudoración excesiva, aumento de tasa cardiaca, etcétera) y del sistema nervioso somático (tensión muscular), aunque también se activan otros sistemas (hormonal, inmune, etcétera). Por su parte, la ansiedad situacional predominante entre los pacientes del grupo de obesidad fue la ansiedad fóbica, en la cual se agrupan situaciones potencialmente fóbicas (tabla 3).

Tabla 2.

Respuestas de ansiedad con mayor frecuencia en pacientes con obesidad

\begin{tabular}{lcccc} 
& ModA & DT & Mínimo & MÁxımo \\
\hline Ansiedad fisiológica & 85.0 & 37.33 & 5 & 99 \\
Ansiedad motora & 5.0 & 34.87 & 5 & 99 \\
$\begin{array}{l}\text { Ansiedad cognitiva } \\
\text { Fuente: Elaboración propia, 2017. }\end{array}$ & 58.37 & 5 & 99 \\
\hline
\end{tabular}

Fuente: Elaboración propia, 2017.

Tabla 3.

Situaciones de ansiedad con mayor frecuencia en pacientes con obesidad

\begin{tabular}{lcccc} 
& ModA & DT & Mínimo & MÁximo \\
\hline $\begin{array}{l}\text { Ansiedad de } \\
\text { evaluación }\end{array}$ & 5 & 35.31 & 5 & 95 \\
$\begin{array}{l}\text { Ansiedad } \\
\text { interpersonal }\end{array}$ & 15 & 35.57 & 5 & 95 \\
\hline $\begin{array}{l}\text { Ansiedad fóbica } \\
\begin{array}{l}\text { Ansiedad de la vida } \\
\text { cotidiana }\end{array}\end{array}$ & 20 & 35.79 & 10 & 95 \\
\hline $\begin{array}{l}\text { Fuente: Elaboración propia } \\
\text {, 2017 }\end{array}$ & 5 & 45.36 & 5 & 95 \\
\hline
\end{tabular}

Fuente: Elaboración propia, 2017.

\section{Faceta de mindfulness predominante en pacientes con obesidad morbida}

En el análisis de las facetas de mindfulness, la faceta que se encontró con mayor frecuencia fue la no reactividad ante las experiencias que se refiere a la medida en que se atiende a las experiencias internas (como pensamientos y emociones), sin participar en respuestas automáticas (como comer en exceso y beber); la de menor presencia dentro del grupo fue la de describir, que se refiere a la capacidad de poner en palabras las experiencias, pero sin establecer un juicio ni análisis conceptual acerca de ellas (tabla 4).

\section{Relación entre la ansiedad general y los niveles de mindfulness en pacientes con obesidad mórbida}

Utilizando el estadístico no paramétrico de Spearman rho se encontró que hay una importante correlación negativa entre ambas variables; esto es, cuando hay puntuaciones de facetas de mindfulness más altas se presentan niveles de ansiedad bajos ( $r h o=-0.860 ; p=0.013$ ).

\section{Tabla 4.}

Facetas de mindfulness

\begin{tabular}{|c|c|c|c|c|}
\hline & MOdA & DT & Mínimo & MÁxIMo \\
\hline Observación & 53 & 11.89 & 53 & 80 \\
\hline Describir & 25 & 24.72 & 25 & 83 \\
\hline Actuar con conciencia & 65 & 13.68 & 40 & 83 \\
\hline $\begin{array}{l}\text { No reactividad ante } \\
\text { las experiencias }\end{array}$ & 74.3 & 13.21 & 42.9 & 74.3 \\
\hline $\begin{array}{l}\text { No juicio ante las } \\
\text { experiencias } \\
\text { Euente: Elaboración pro }\end{array}$ & $\begin{array}{l}40 \\
2017\end{array}$ & 17.48 & 32.5 & 75 \\
\hline
\end{tabular}

\section{Relación entre las situaciones y respuestas de ansiedad y las facetas mindfulness en pacientes con obesidad mórbida}

Al relacionar las cinco facetas mindfulness con las respuestas de ansiedad se obtuvo una importante correlación negativa significativa entre el factor de mindfulness no reactividad ante las experiencias y la ansiedad del tipo motora (rho $=-0.936 ; p=0.002)$. La no reactividad ante las experiencias se refiere a la tendencia de dejar pasar los pensamientos y los sentimientos, sin quedar atascados ni dejarse llevar por ellos; cuando esta faceta es mayor, la ansiedad de tipo motor es menor, no presentándose relación significativa en ninguna de las otras fases con los tipos de ansiedad (tabla 5)

Tabla 5.

Relación entre las cinco facetas y las respuestas de ansiedad

\begin{tabular}{|c|c|c|c|c|c|}
\hline & 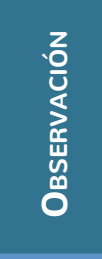 & 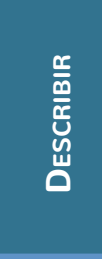 & 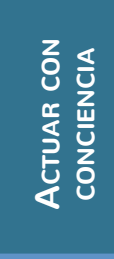 & 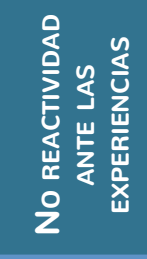 & 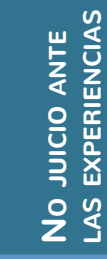 \\
\hline $\begin{array}{l}\text { Ansiedad } \\
\text { fisiológica }\end{array}$ & 0.528 & 0.064 & -0.560 & -0.743 & -0.415 \\
\hline $\begin{array}{l}\text { Ansiedad } \\
\text { motora }\end{array}$ & 0.547 & 0.514 & -0.202 & $-0.936 * *$ & 0.094 \\
\hline $\begin{array}{l}\text { Ansiedad } \\
\text { cognitiva }\end{array}$ & 0.262 & 0.264 & 0.245 & -0.718 & 0.093 \\
\hline
\end{tabular}

Nota: * La correlación de Spearman es significativa al nivel

0.05 (bilateral). ${ }^{* *}$ La correlación de Spearman es significativa al nivel 0.01 (bilateral).

Fuente: Elaboración propia, 2017.

$\mathrm{Al}$ analizar las cinco facetas mindfulness con las cuatro áreas de situaciones ansiógenas en pacientes con obesidad del HRAEV (tabla 6), se encontró de nuevo que la variable con relaciones fuertes de manera nega- 
tiva significativa con varias situaciones de ansiedad es la no reactividad ante las experiencias, donde a mayor no reactividad ante las experiencias menor cantidad de situaciones de ansiedad de evaluación ( $r$ ho $=-0.918 ; p$ $=0.004)$; menor ansiedad interpersonal (rho $=-0.973$; $\mathrm{p}=0.000)$ y menor ansiedad de la vida cotidiana ( $r$ ho $=$ $-0.872 ; p=0.011)$.

\section{Tabla 6.}

Relación entre las 5 facetas y las situaciones de ansiedad.

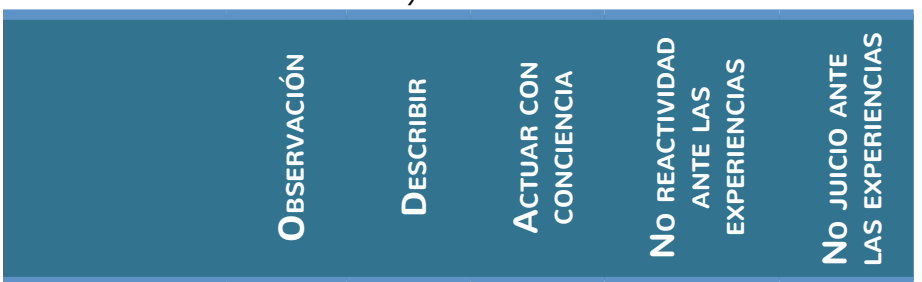

\begin{tabular}{|c|c|c|c|c|c|}
\hline $\begin{array}{l}\text { Ansiedad de } \\
\text { evaluación }\end{array}$ & 0.673 & 0.282 & -0.209 & $-0.918^{* *}$ & -0.206 \\
\hline $\begin{array}{l}\text { Ansiedad } \\
\text { interpersonal }\end{array}$ & 0.748 & 0.445 & -0.300 & $-0.973^{* *}$ & -0.093 \\
\hline $\begin{array}{l}\text { Ansiedad } \\
\text { fóbica }\end{array}$ & 0.150 & 0.209 & -0.064 & -0.664 & 0.019 \\
\hline $\begin{array}{l}\text { Ansiedad } \\
\text { de la vida } \\
\text { cotidiana }\end{array}$ & 0.472 & 0.321 & -0.009 & $-0.872^{*}$ & -0.019 \\
\hline \multicolumn{6}{|c|}{$\begin{array}{l}\text { Nota: }{ }^{*} \text { La correlación de Spearman es significativa al nivel } \\
0.05 \text { (bilateral). }{ }^{* *} \text { La correlación de Spearman es significativa } \\
\text { al nivel } 0.01 \text { (bilateral). } \\
\text { Fuente: Elaboración propia, } 2017 .\end{array}$} \\
\hline \multicolumn{6}{|l|}{ Discusión } \\
\hline
\end{tabular}

El objetivo de este estudio fue explorar la relación que se presentaba en los entre las facetas de mindfulness y las situaciones y respuestas de ansiedad en una población clínica de obesidad.

En la población analizada se encontró que la ansiedad se presentaba sobre todo por medio de respuestas de carácter fisiológico, las cual hacen referencia a los cambios corporales, y que son predominantemente generadas por situaciones del tipo fóbicas.

Por otra parte, en el análisis de la presencia de las facetas de mindfulness en los pacientes con obesidad se observó que la que se muestra con menor presencia es el describir; algunos estudios han relacionado los bajos niveles de esta faceta con problemas presentes en la obesidad (Levin, Dalrymple, Himes y Zimmermana, 2014; Lattimore, Fisher y Malinowski, 2011) donde se encuentra una relación entre los déficit de la faceta de describir y la presencia de trastornos alimenticios (como el atracón y la alimentación emocional).

Para comenzar a analizar la relación entre las situaciones y respuestas de ansiedad y las facetas del mindfulness en nuestra población de obesidad, primero buscamos si dentro de esta población había relación entre las variables de ansiedad general y los niveles de mindfulness; los resultados indicaron que cuando hay un nivel de mindfulness más alto, se observan niveles de ansiedad más bajos en los pacientes de obesidad mórbida; esto coincide con los resultados de Pintado-CucareIla y Rodríguez-Salgado (2016), donde se encontró que las pacientes con obesidad que tienen menos niveles de mindfulness por lo general suelen estar más ansiosos.

A la vez se buscó analizar si las relaciones entre las facetas de mindfulness y las respuestas y situaciones de ansiedad en una población clínica con obesidad mórbida se relacionaban de la misma manera en que se había relacionado la ansiedad y el mindfulness en una población no clínica, donde la faceta "no juzgar" presentaba mayor relación (Tomlinson et al., 2017; Medvedev et al., 2018).

Nuestros resultados muestran que la única faceta de mindfulness con una relación negativa fuerte y significativa con las respuestas y las situaciones de ansiedad es la no reactividad ante las experiencias, tanto con la respuesta de ansiedad motora como con las situaciones de ansiedad de evaluación, interpersonal y de la vida cotidiana; estos resultados muestran una discrepancia con las relaciones encontradas antes en poblaciones no clínicas, donde los resultados indicaban que niveles más bajos de autojuicio resultaban en puntuaciones más bajas de ansiedad (Tomlinson et al., 2017; Medvedev et al., 2018).

Por otra parte, nuestros resultados sugieren que la faceta "no reactividad", que se refiere a la medida en que se atiende a las experiencias internas sin participar en respuestas automáticas, es la que muestra mayor relación con las respuestas y las situaciones de ansiedad.

Este resultado se suma a la evidencia de que sólo las facetas "actuar con conciencia", "no juzgar" y "no reactividad" pueden ser predictores significativos de síntomas psicológicos (Baer et al., 2006); en este estudio con pacientes con obesidad mórbida, la faceta "no juzgar" no fue el factor que más se relacionaba con la ansiedad (Medvedev et al., 2018), sino que la que mayor se relaciona con la respuesta de ansiedad motora y las situaciones ansiógenas de evaluación, interpersonales y de la vida cotidiana es la "no reactividad".

Una explicación a esta diferencia pueden ser los niveles de peso en la población estudiada ya que se había encontrado que sólo las facetas de "no reactividad" y "observación" se asociaban de modo inverso con el estado del peso, esto es, entre mayores sean los niveles de peso hay menos capacidad para atender las experiencias sin reaccionar de manera automática (Camilleri, Méjean, Bellisle, Hercberg y Péneau, 2015), porque las personas 
con obesidad mórbida manejan niveles de peso muy elevados; el autojuicio puede no ser tan significativo, siendo "no reactividad" la faceta con mayor deficiencia, y por tanto la que más contribuya a la presencia de distintas patologías emocionales, incluyendo la ansiedad.

Varios estudios indican que deficiencia en la faceta de no reactividad suele presentarse a través de trastornos alimenticios que involucran la pérdida de control sobre la alimentación como el comer emocional, comer externo y trastorno de atracón entre otros (Lavender, Gratz y Tull, 2011; Jablonski, 2013; Sala y Levinson, 2017), por lo que dentro de la obesidad las técnicas para fomentar la no reactividad podrían conducir a una autorregulación efectiva a través de la modulación del comportamiento automático, que por lo tanto puede resultar en disminuir las preocupaciones que les pueden estar generando mayor ansiedad.

Con estos resultados nos podemos cuestionar si en la población estudiada la ansiedad se genera sobre todo como consecuencia de los comportamientos automáticos que propician el comer en exceso, más que como consecuencia de los autojuicios negativos, como en las poblaciones no clínicas.

Al igual que concluir que, de acuerdo con nuestros resultados, estas relaciones se pueden presentar de manera distinta, mas los resultados de este estudio deben interpretarse con precaución dadas sus múltiples limitaciones.

\section{Conclusiones}

Nuestros resultados sugieren que la relación de las facetas de mindfulness con las situaciones y respuestas de ansiedad se presenta de manera distinta en poblaciones con obesidad mórbida, porque en una población no clínica suele tener mayores niveles de relación la faceta "no juzgar", mientras que en nuestra población clínica de obesidad muestra mayor relación la faceta "no reactividad".

Podemos concluir que la función beneficiosa de las facetas de atención plena en los síntomas de ansiedad no puede generalizarse de poblaciones sin obesidad a poblaciones con obesidad, porque los factores generadores de ansiedad en pacientes clínicos con obesidad pueden estar relacionados más con problemas de modulación en el comportamiento automático que con pensamientos negativos de sí mismos, debido a la naturaleza de su patología.

Por tanto, se recomienda dar mayor importancia a la faceta "no reactividad" dentro de las intervenciones que utilicen mindfulness con esta población.
Si se quiere replicar la siguiente investigación, es recomendable que se utilice una muestra más grande, para dar mayor relevancia a los resultados.

A partir de este trabajo, hay diferentes líneas de investigación que quedan abiertas, como llevar los resultados a un enfoque más práctico implementando técnicas que utilicen el mindfulness como base, en una población que presente obesidad. De igual manera es recomendable que se investiguen las conductas motoras específicas con que mindfulness puede estar relacionada, como el trastorno de atracón o el comer emocional.

\section{Referencias}

Baer, R., Hopkins, J., Krietemeyer, J., Smith, G., y Toney, I. (2006). Using self-report assessment methods to explore facets of Mindfulness. Assessment [archivo pdf], 13, 27-45. Recuperado de: http://www.ncbi.nlm.nih.gov/ pubmed/16443717

Barrera, A., Rodríguez, G., \& Molina, M. (2013). Escenario actual de la obesidad en México. Temas de actualidad. Recuperado de http://www.medigraphic.com/pdfs/imss/ im-2013/im133k.pdf

Bishop, R., Lau, M., Shapiro, S., Carlson, L., Anderson, N., Carmody, J., Segal, Z., Abbey, S., Speca, M., Velting, D., \& Devins, G. (2004). Mindfulness: A Proposed Operational Definition [archivo pdf]. Clinical Psychology: Science and Practice, 11(3), pp. 230-241. Recuperado de: http://www. jimhopper.com/pdfs/bishop2004.pdf

Camilleri, G., Méjean, C., Bellisle, F., Hercberg, S., y Péneau, S. (2015). Association between Mindfulness and Weight Status in a General Population from the NutriNet-Santé Study. PloS one, 10(6). doi:10.1371/journal.pone.0127447

Cano-Vindel, A., Fernández, J., \& Spielberger, C. (2012). The experience and expression of anger and anxiety in bronchial asthma patients. Ansiedad y Estrés, 18, 221-230.

Cano-Vindel, A., Miguel-Tobal, J.J., Gonzales, H., \& Iruarrizaga, I. (1994). El afrontamiento de la ansiedad en las drogodependencias. Anales de Psicología, 10(2), pp. 145-156.

Endler, N. S. (1981). Person-Situation Interaction and Anxiety. En I. L. Kutash, L. B.

Holzel, B., Lazar, S., Gard, T., Schuman-Olivier, Z., Vago, D., \& Ott, U. (2011). How Does mindfulness meditation work? Proposing mechanisms of action from a conceptual and neural perspective. Perspectives on Psychological Science, 6, pp. 537-559, doi: 10.1177/1745691611419671

Jablonski, M. (2013). The relationship between dispositional mindfulness and eating: an analysis of self-reported and in vivo eating behaviors in undergraduate females. Electronic Theses and Dissertations. doi: 10.18297/ etd/666.

Kabat-Zinn, J., (1990). Principles of mindfulness practice. Full catastrophe living. New York. Recuperado de: https:// clinic.psy.uq.edu.au/pdf/mindfulness-practice-article-jonkabatzinn.pdf

Keng, S., Smoski, M., \& Robins, C. (2011). Effects of mindfulness 
on psychological health: A review of empirical studies. Clinical Psychology Review, 31, pp. 1041-1056. doi: 10.1016/j.cpr.2011.04.006

Kocovski, N. L., Segal, Z. V., Battista, S. R., \& Didonna, F. (2009). Mindfulness and psychopathology: Problem formulation, Clinical handbook of mindfulness (pp. 85-98). Nueva York: Springer.

Lattimore, P., Fisher, N., \& Malinowski, P. (2011). A cross-sectional investigation of trait disinhibition and its association with mindfulness and impulsivity. Appetite, 56, 241-248.

Lavender, J., Gratz, K., \& Tull, M. (2011). Exploring the relationship between facets of mindfulness and eating pathology in women. Cognitive Behaviour Therapy, 40(3), 174-182. Recuperado de: https://doi.org/10.1080/16506073.2011. 555485

Levin, M., Dalrymple, K., Himes, S., \& Zimmermana, M. (2014). Which facets of mindfulness are related to problematic eating among patients seeking bariatric surgery?. Eating Behaviors, 15, 298-305.

Meda, R., Herrero, M., Blanco, L., Moreno-Jimenez, B., \& Palomera, A. (2015). Propiedades psicométricas del "cuestionario de cinco facetas de la conciencia plena" (five facet mindfulness questionnaire, FFMQ-M) en México. Psicología Conductual, 23(3) pp. 467-487.

Medvedev, O., Norden, P., Krägeloh, C., \& Siegert, R. (2018). Investigating Unique Contributions of Dispositional Mindfulness Facets to Depression, Anxiety, and Stress in General and Student Populations. Mindfulness. doi:10.1007/s12671-018-0917-0.

Montero, I., \& León, O. G. (2007). A guide for naming research studies in Psychology. International Journal of Clinical and Health Psychology, 7(3), 847-862. Recuperado de: http:// www.redalyc.org/articulo.oa?id $=33770318$

Organización Mundial de la Salud (OMS) (2014). Obesidad y sobrepeso. Nota descriptiva, 311. Recuperado de: http:// www.who.int/mediacentre/factsheets/fs311/es/index.html
Pintado-Cucarella, C., \& Rodríguez-Salgado, P. (2016). Mindful eating and its relationship with body mass index, binge eating, anxiety and negative affect. Journal of Behavior, Health \& Social Issues, 8(2), 1-42. doi: 10.1016/j. jbhsi.2016.11.003.

Rogers, J., Ferrari, M., Mosely, K., Lang, C., \& Brennan, L. (2016). Mindfulness-based interventions for adults who are overweight or obese: a meta-analysis of physical and psychological health outcomes. Obesity reviews, 18(1). doi: https://doi.org/10.1111/obr.12461.

Sala, M., \& Levinson, C. A. (2017). A Longitudinal Study on the Association Between Facets of Mindfulness and Disinhibited Eating. Mindfulness, 8(4), 893-902. doi: 10.1007/s12671-016-0663-0.

Schmidt, C., \& Vinet, E. (2015). Atención plena: Validación del Five Facet Mindfulness Questionnaire (FFMQ) en estudiantes universitarios chilenos. Terapia psicológica, 33(2), 93-102. doi: https://dx.doi.org/10.4067/S071848082015000200004.

Secretaría de Salud (2012). Prevención, diagnóstico y tratamiento del sobrepeso y la obesidad exógena. Cenetec, México. Recueprado de: http://www.cenetec. salud.gob.mx/descargas/gpc/CatalogoMaestro/046 GPC ObesidadAdulto/IMSS 046_08_EyR.pdf

Teasdale, J. D., Segal, Z. V., \& Williams, J. M. G. (2003). Mindfulness training and problem formulation. Clinical Psychology: Science and Practice, 10, 157-160.

Tobal, M., \& Vindel, C. (1999). Evaluación de la ansiedad desde un enfoque interactivo y multidimensional: El inventario de situaciones y respuestas de ansiedad (ISRA). Psicología Contemporánea, 6(1), pp. 14-21.

Tomlinson, E., Yousaf, O., Vittersø, A., \& Jones, L. (2017). Dispositional mindfulness and psychological health: a systematic review. Mindfulness. doi: https://doi. org/10.1007/s12671-017-0762-6. 


\section{Meta-Análisis del Artículo}




\section{Perfil de Evaluación entre pares}
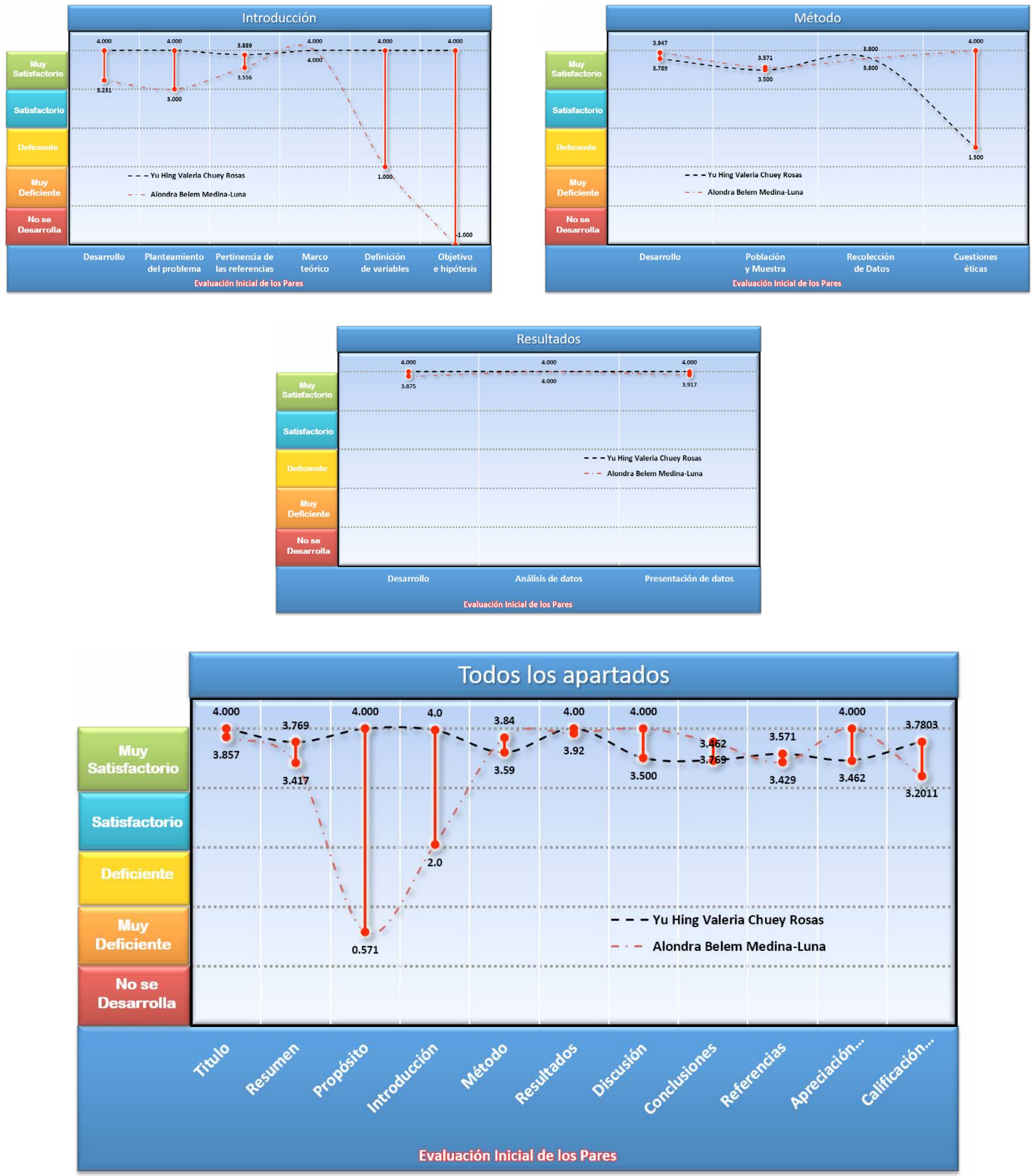


\section{Índice de Concordancia}

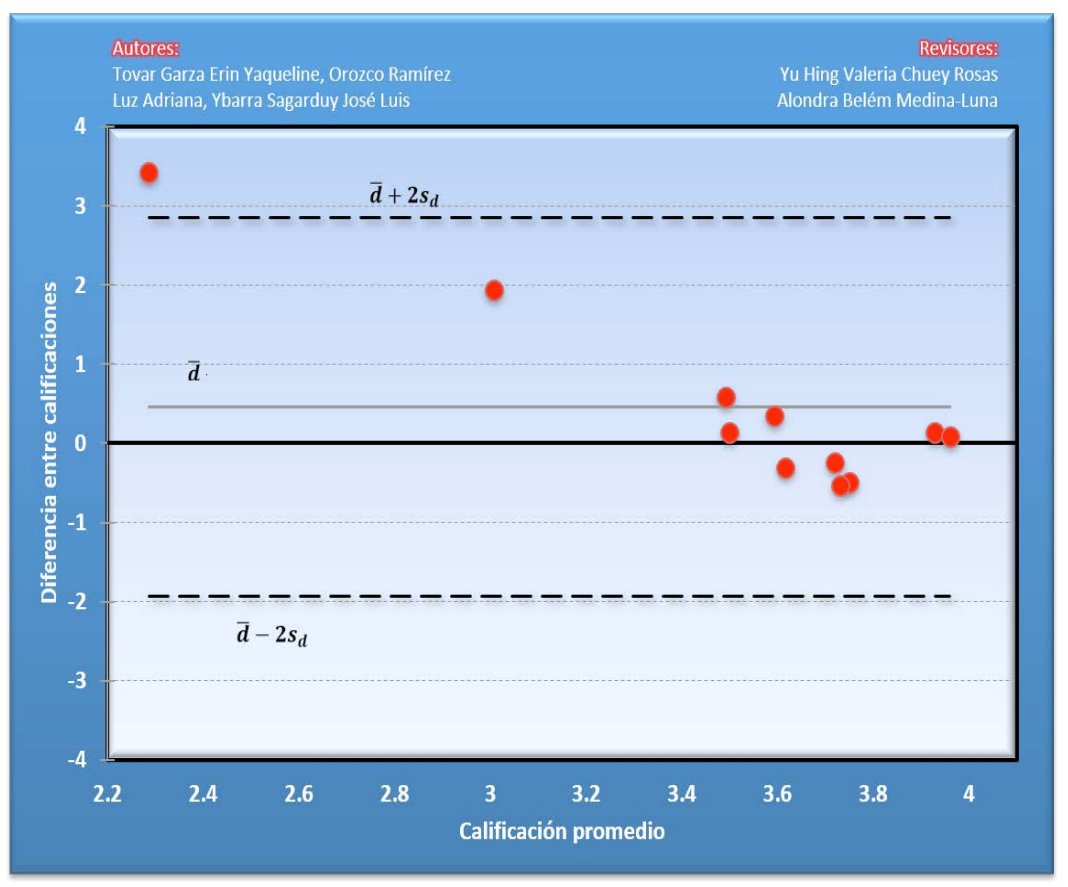

Índice de Acuerdo

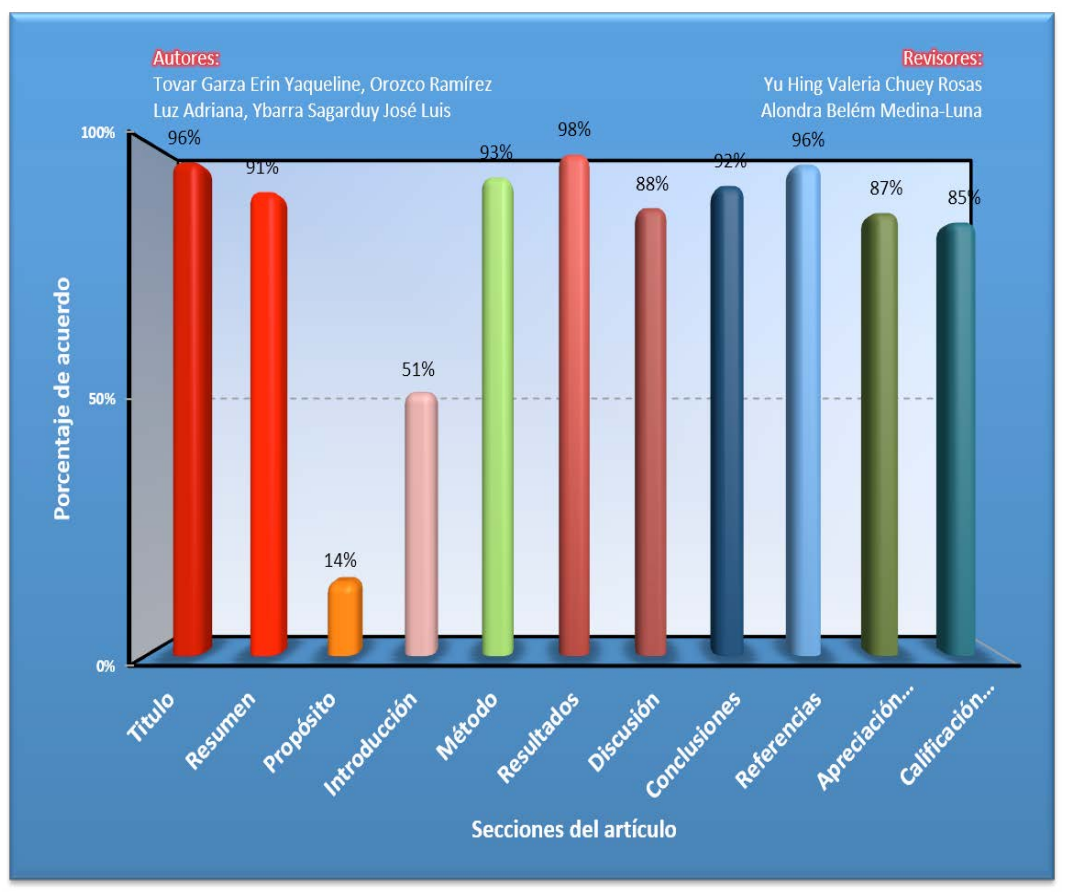




\begin{tabular}{|c|c|}
\hline Revisor 1 & Revisor 2 \\
\hline Yu Hing Valeria Chuey Rosas & Alondra Belem Medina Luna \\
\hline \multicolumn{2}{|c|}{ Título/Autoría } \\
\hline El título es correcto respecto a la investigación. & $\begin{array}{l}\text { Aparece la abreviatura de Ciudad (Cd.) Se incluyen gra- } \\
\text { dos académicos y se especifica no incluírlos }\end{array}$ \\
\hline \multicolumn{2}{|c|}{ Resumen } \\
\hline Agregar el resultado con valor numérico. & $\begin{array}{l}\text { Se encuentran las descripciones solicitadas pero en } \\
\text { parrafos desafasados. No se presentan resultados con } \\
\text { valore numéricos. Se utiliza abreviatura en la palabra } \\
\text { Ciudad (Cd.) Rebasa el límite de palabras sugeridas } \\
\text { Solo menciona } 4 \text { palabras clave }\end{array}$ \\
\hline \multicolumn{2}{|c|}{ Próposito del Estudio } \\
\hline $\begin{array}{l}\text { El propósito del estudio es claro, que es explorar ciertas } \\
\text { asociaciones. }\end{array}$ & $\begin{array}{l}\text { No se presentan preguntas de investigación o hipótesis } \\
\text { del propósito del estudio que puedan ser directrices para } \\
\text { entender lo que se busca con el presente estudio, desa- } \\
\text { rrollar preguntas o una hipótesis general de manera clara } \\
\text { y concisa aportaría a entender mejor el trabajo propuesto }\end{array}$ \\
\hline \multicolumn{2}{|c|}{ Introducción } \\
\hline $\begin{array}{l}\text { Sería pertinente que se señale que en nuestro país es } \\
\text { un tema (por ejemplo el -mindfulness-) que cada vez } \\
\text { se estudia más, pero que es necesario seguir generando } \\
\text { información al respecto. }\end{array}$ & $\begin{array}{l}\text { Es importante desarrollar las hipótesis, definirlas y vin- } \\
\text { cularlas para establecer claramente el estudio de investi- } \\
\text { gación en estos aspectos, así mismo es pertinente definir } \\
\text { claramente las variables involucradas en el estudio. }\end{array}$ \\
\hline
\end{tabular}


Artículo Empírico | Mindfulness y ansiedad en pacientes...| Tovar-Garza, Orozco-Ramírez e Ybarra-Sagarduy

\begin{tabular}{|c|c|}
\hline Revisor 1 & Revisor 2 \\
\hline \multicolumn{2}{|c|}{ Método } \\
\hline Faltaría la sección de mediciones como tal. & $\begin{array}{l}\text { Es importante describir la validez y confiabilidad de am- } \\
\text { bos instrumentos utilizados }\end{array}$ \\
\hline \multicolumn{2}{|c|}{ Resultados } \\
\hline $\begin{array}{l}\text { Agregar unidad de medida a algunos datos sociodemo- } \\
\text { gráficos. }\end{array}$ & $\begin{array}{l}\text { Al establecer claramente las hipótesis y variables desde } \\
\text { el inicio del documento será mas facil entender los re- } \\
\text { sultados y mejorar la puntuación en los reactivos de esta } \\
\text { faceta de la evaluación del manuscrito completo. }\end{array}$ \\
\hline \multicolumn{2}{|c|}{ Discusión } \\
\hline $\begin{array}{l}\text { Revisar el uso de comas y palabras pegadas. Señalar las } \\
\text { limitaciones del estudio. }\end{array}$ & Bien \\
\hline \multicolumn{2}{|c|}{ Conclusiones } \\
\hline $\begin{array}{l}\text { Sugerencia: entrecomillar las facetas para que se distin- } \\
\text { gan en la narración de las conclusiones. }\end{array}$ & $\begin{array}{l}\text { Ampliar las conclusiones mencionando la significancia } \\
\text { estadística hallada }\end{array}$ \\
\hline \multicolumn{2}{|c|}{ Referencias } \\
\hline Revisar palabras pegadas. & $\begin{array}{l}\text { Las referencias utilizadas deben ser más actuales, hay pocas } \\
\text { de los últimos } 5 \text { años a la fecha en que se realizó el estudio }\end{array}$ \\
\hline
\end{tabular}




\section{Historia del Proceso}

\section{EDITORIAL}

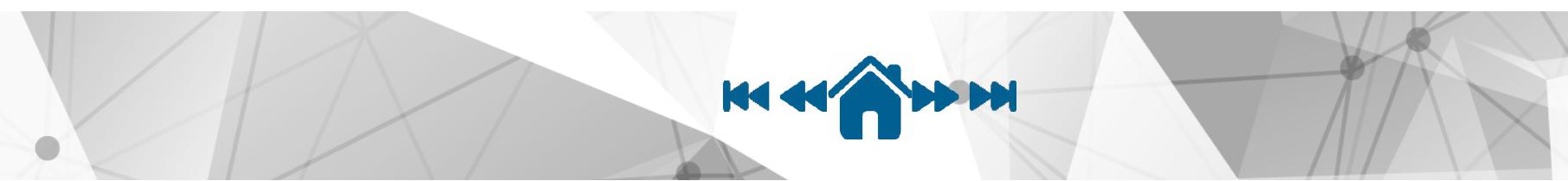

